Upcoming: December 23-24 15th MRS-J Academic Symposium, MRS-J.

To list an event in the Calendar, contact J. Meiksin, Materials Research Society, 506 Keystone Drive, Warrendale, PA 150867573; 724-779-3004 ext. 522; fax 724-779-8313; bulletin@mrs.org.

MRS, A-MRS, B-MRS, C-MRS, E-MRS, MRS-A, MRS-I, MRSJ, MRS-K, MRS-R, MRS-S, MRS-T, M-MRS, or IUMRS at the end of an entry indicates sponsorship or co-sponsorship of an event by the International Union of Materials Research Societies or one of its adhering bodies. "Endorsed" identifies events endorsed by MRS.

$\nabla$ identifies a new or revised entry this month.

See the September 2004 MRS BULLETIN for November 2004 Calendar entries. For updates, access www.mrs.org/ meetings/other/.

\section{DECEMBER 2004}

2 SPE Rapid Design

Engineering, and Moldmaking Conf.: Reduce Time to Market, Frankfurt, Germany. Yetty Pauwels, Society of Plastics Engineers, Bistkapellei 44, Antwerp, Belgium, 32-3-541-77-55; fax 32-3-541-84-25; e-mail spe.europe@pi.be; www.4spe.org/semconf/ conferences/conferences.htm.
6-8 Intl. Symp. on Advanced Materials and Processing, Kharagpur, India. A.K. Banthia, Indian Institute of Technology, Kharagpur, India, 91-3222-282274; e-mail akb@matsc.ittkgp.ernet.in; www.iitkgp.ernet.in/departments/ home.php?deptcode=MS.

9-11 IEEE Semiconductor Interface Specialists Conf., San Diego, CA. Karen Kuiper, 6446 Tucker Avenue, McLean, VA 22101; 703-660-0913; e-mail kjkuiper@mindspring.com; www. ieeesisc.com.

\section{3-24 $\nabla$ 15th MRS-J Academic} Symposium, Suruga-dai, Japan. Secretariat, 3-2-1 Sakato, Takatsuku, Kawasaki, Kanagawa 213-0012, Kanagawa Science Park, West Building 413-F, Japan; tel./fax 044829-1782; e-mail mrsj@ksp.or.jp; www.mrs-j.org. MRS-J.

\section{JANUARY 2005}

4-8 $\nabla$ Optical Probes, Bangalore, India. Chandrabhas Narayana,

Indian Institute of Science,

Bangalore, India, e-mail

op2005@jncasr.ac.in;

www.jncasr.ac.in/op2005.

10-14 IUVSTA Workshop on One-Dimensional Nanomaterials, Taipei, Taiwan. R. Chu-Chen, 886-2-33665202; fax 886-223655404; e-mail iuvsta@ms. ntu-ccms.ntu.edu.tw.
13-15 $\nabla$ UNESCO-Physics for Tomorrow, Paris, France. E-mail launch@wyp2005.org; www. nano2005.imr.edu/english index.html.

17-21 $\nabla$ 5th Intl. Conf. on Electroluminescence of Molecular Materials and Related Phenamena (ICEL-5), Phoenix, Arizona. Prof. Ghassan E. Jabbour, ICEL-5, Department of Chemical and Materials Engineering, Ira A. Fulton School of Engineering, Arizona State University, P.O. Box 876006 Tempe, AZ 85287-6006 USA; 480-727-8930, 480-727-7002 (main campus); fax 480-727-8957; e-mail Jabbour@asu.edu; www.public.asu.edu/ yyoshiok ICEL\%205/index.htm.

22-27 Photonics West 2005, San Jose, CA. SPIE, SPIE Meetings, P. O. Box 10, Bellingham, WA 98227-0010; 360-676-3290; fax 360-647-1445; e-mail spie@spie.org; spie.org/Conferences/calls/05/pw.

\section{FEBRUARY 2005}

6-10 $\nabla$ AMN: Intl. Conf. on Advanced Materials and Nanotechnology, Queenstown, New Zealand. Conference Secretariat, Conference Office, Centre for Continuing Education, University of Canterbury, Private Bag 4800, Christchurch, New Zealand; 64-3-364-2534; fax 64-3364-2324; e-mail amn2@cont. canterbury.ac.nz; www.elec. canterbury.ac.nz/AMN-2. Endorsed.
6-11 Second Intl. Conf. on Advanced Materials and Nanotechnology (AMN-2), Queenstown, New Zealand. Conference Secretariat, University of Canterbury, Private Bag 4800 ,

Christchurch, New Zealand 64-3-364-2534; fax 64-4-364-2324 e-mail amn2@cont.canterbury.ac.nz; www.macdiarmid.ac.nz/amn-2.

10-12 $\nabla$ 16th Annual Genera Meeting of MRS-India, Pune, India. Dr. K.Vijayamohanan, Convenor, 16th AGM-MRSI Secretariat, Physical and Materials Chemistry Division, National Chemical Laboratory, Pune-411 008 INDIA; 020-25893300 ext.2002/2276; fax 020-25893044; e-mail mrsi2005@dalton.ncl.res.in; www.igcar.ernet.in/mrsi. MRS-I.

16-18 『 Smart Coatings 2005 Orlando, FL. Sandra Tanner Coatings Research Institute 430 W. Forest Ave., Ypsilanti, MI, 48197: 734-487-2203; fax 734483-0085; e-mail sandy.tanner@ emich.edu; www.emich.edu/public/ coatings research/smartcoatings/ index2.html.

\section{MARCH 2005}

6-9 Materials for Advanced Metallization 2005 (MAM2005), Dresden, Germany. MAM2005, 49-371-531-3130; e-mail info@ mam-conference.org; www. mam-conference.org.
6-10 SPIE-Smart Structures and Materials \& Nondestructive Evaluation for Health Monitoring and Diagnostics, San Diego, CA. SPIE, SPIE Meetings, P.0. Box 10, Bellingham, WA, 98227-0010; 360-676-3290; fax 360-647-1445; e-mail spie@spie.org; spie.org/events/ssnde.

8-9 $\boldsymbol{\nabla}$ RAPRA-High Performance Fillers 2005, Cologne, Germany. Rapra Technology Limited, Shawbury, Shrewsbury, SY4 4NR, United Kingdom; 44-0-1939250383; fax 44-0-1939-251118; e-mail conferences@rapra.net; www.polymerconferences.com.

14-18 ASNT Spring Conf. and 14th Annual Research Symp., Albuquerque, NM. Kelly Thomas, American Society for Nondestructive Testing, P.O. Box 28518, 1711 Arlingate Ln, Columbus, $\mathrm{OH} 43228$ 0518, 800-222-2768 x227; fax 614274-6899; kthomas@asnt.org; http://www.asnt.org.

15-16 \ RAPRA-Multimaterial Polymer Structures Conf., Stuttgart Germany. Rapra Technology Limited, Shawbury, Shrewsbury, SY4 4NR, United Kingdom; 44-0 1939-250383; fax 44-0-1939-251118; e-mail conferences@rapra.net; www.polymerconferences.com.

21-25 2005 American Physical Society March Meeting, Los Angeles, CA. Terri Gaier, APS, fax 301-2090866; e-mail gaier@aps.org; http://www.aps.org/meet/MAR05

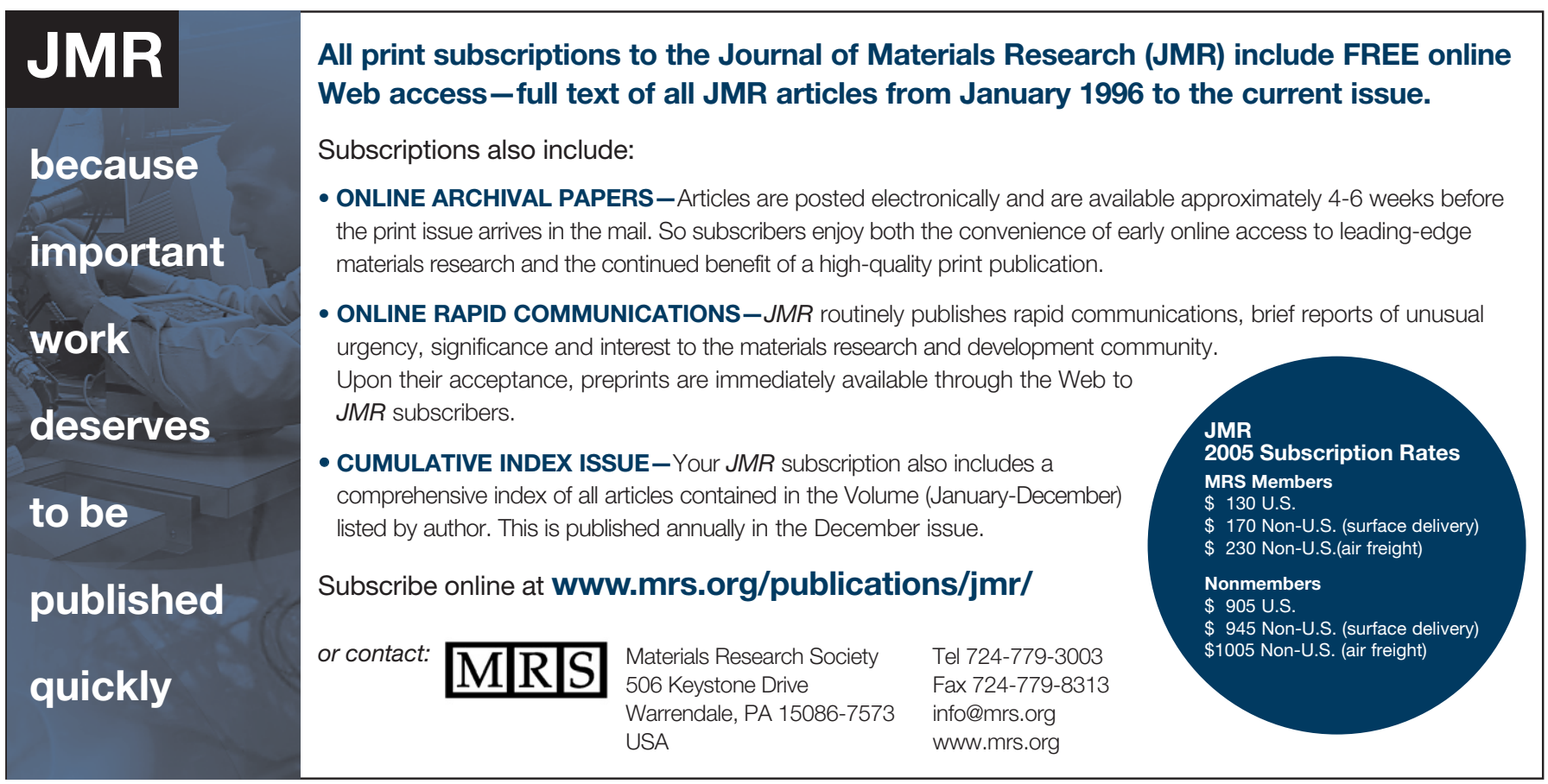


28-1 MRS Spring Meeting, San Francisco, CA. Materials Research Society, 506 Keystone Dr., Warrendale, PA 15086-7573; 724779-3003; fax 724-779-8313; e-mail info@mrs. org; www.mrs.org. MRS

\section{APRIL 2005}

4-5 OPTO-Ireland 2005, Dublin Ireland. SPIE, SPIE Meetings, P.O Box 10, Bellingham, WA, 982270010; 360-676-3290; fax 360-647 1445; e-mail spie@spie.org; spie.org/conferences/calls/05/ire

4-6 ₹ PM Asia-2005, Shanghai, China. Nick Williams, Inovar Event Management, Dogpole House, Shrewsbury, United Kingdom, 44-0-1743-241289; e-mail Nick@inovar-events.com; www.pmasia2005.com.

4-8 2005 Intl. Magnetics Conf. (INTERMAG2005), Nagoya, Japan. Intermag 2005 Secretariat, c/o Convention Linkage, Inc., AkasakaNihon Bldg., 9-5-24, Akasaka, Minato-ku, Tokyo 107-0052, Japan; 81-3-5770-5531; fax 81-3-57705532; e-mail info@intermag2005.jp; www.intermag2005.jp. Endorsed.

11-13 The Intl. Conf. on Ceramic Interconnect and Ceramic Microsystems Technology, Westerville, OH. Ann Bell, IMAPS, 202-548-8717; e-mail abell@imaps. org; www.ceramics.org

11-14 Fourteenth Intl. Conf. on Microscopy of Semiconducting Materials, Oxford, United Kingdom. Univ. of Sheffield, Tony Cullis, Sheffield, United Kingdom, 44-114-222-5407: fax 44-114272-6391; e-mail a.g.cullis@ sheffield.ac.uk; www.rms.org.uk Endorsed.
24-28 15th Intl. Conf. on Wear of Materials, San Diego, CA. Gill Heaton, Secretariat, Hillside Cottages, Wheatley Road, Islip, Oxford 0X5 2TF, UK; 44-0-1865373625; fax 44-0-1865-375855; e-mail wom-conference@elsevier. com; www.wom-conference. elsevier.com

\section{MAY 2005}

8-10 $\nabla$ Meeting of Society of Nano Science and Technology, Sendai, Japan. Yukiko Miyahara, Tohoku University, 2-1-1 Katahira Aoba-ku, Sendai, Japan, 81-22 215-2054; fax 81-22-215-2052 e-mail nan02005@imr.edu; www.nano2005.imr.edu/ english index.html.

10-11 $\nabla$ RAPRA-Blowing Agents \& Foaming Processes 2005, Stuttgart, Germany. Rapra Technology Limited, Shawbury, Shrewsbury, SY4 4NR, United Kingdom, 44-0-1939-250383; ax 44-0-1939-251118. e-mail conferences@rapra.net; www.polymerconferences.com.

29-3 2nd Intl. Conf. on Electrophoretic Deposition: Fundamentals and Applications, Barga, Italy. Impe. C. of Sci, Tech. \& Med., A Boccaccini, London, United Kingdom, 44-207-5946731; fax 44-207-5843194

e-mail a.boccaccini@imperial.ac.uk www.engconfintl.org/5ad.html.

29-3 $\nabla$ Solid-Solid Phase Transformations in Inorganic Materials 2005, Phoenix, Arizona. TMS Meeting Services, 184 Thorn Hill Road, Warrendale, PA 15086 USA; 724-776-9000, ext. 243; e-mail mtgserv@tms.org; www. tms.org/meetings/specialty/ptm home.html. Endorsed.
31-3 $\nabla 2005$ E-MRS Spring Meeting, Strasbourg, France. Paul Siffert, General Secretary, European Materials Research Society Headquarters, BP 20, 67037 Strasbourg Cedex 2, France; 33-388-106273; fax 33-3-88-106343; e-mail emrs@phase.c-strasbourg.fr www-emrs.c-strasbourg.fr. E-MRS.

\section{JUNE 2005}

26-30 17th University Conf. on Glass Science, University Park PA. Carlo Pantano; www mri.psu. edu/conferences/glassscience.

\section{JULY 2005}

3-8 Intl. Conf. on Materials for Advanced Technologies (ICMAT 2005) and IUMRS-ICAM 2005, Singapore. Mat Research So (Singapore), ICMAT Secretariat, 3 Research Link, Singapore, 65-6874-1975; fax 65-6777-2393; e-mail icmat@ mrs.org.sg; www. mrs.org.sg/meeting_future. html. IUMRS/MRS-S

4-8 Lucerne Fuel Cell Forum 2005, Lucerne, Switzerland. EFCF, Morgenacherstrasse 2F, P.O. Box 99, Oberrohrdorf, Switzerland, 41-56496-7292: fax 41-56-496-441; e-mail forum@efcf.com

10-15 16th Intl. Conf. on Electronic Properties of TwoDimensional Systems (EP2DS-16) Albuquerque, NM. Jerry Simmons, 505-844-8402; e-mail jsimmon@ sandia.gov. Endorsed.

17-22 Intl. Conf. on Solid State Ionics (SSI-15), Baden-Baden, Germany. Ellen Ivers-Tiffee, c/0 Universität Karlsruhe, Kaiserstr. 12, D-76131 Karlsruhe, Germany; 49-721-608-7491; fax 49-721-6087492; e-mail info@ssi-15.net; www.ssi-15.net.
19-23 5th Intl. Conf. on Intelligent Processing and Manufacturing of Materials, Monterey, CA. IPMM 05

www.mining.ubc.ca/ipmm.

\section{AUGUST 2005}

28-2 6th Intl. Conf. on Nitride Semiconductors (ICNS-6), Breme Germany. Sven Einfeldt, University of Bremen, P.0. Box 330440 , Bremen, Germany; 49-421-2187453; fax 49-421-218-4581; e-mail icns6@ifp.uni-bremen.de; www.ifp.uni-bremen.de/icns6.

30-2 $\boldsymbol{\nabla}$ ECSSC X 2005 European Conf. on Solid State Chemistry, Sheffield, United Kingdom. Louise Draycott, University of Sheffield, Sir Robert Hadfield Building, Mappin Street, Sheffield, United Kingdom, 0-114-222-60-27; fax 0-114-222-59-43.

e-mail L.Draycott@sheffield.ac.uk www.shef.ac.uk/materials/ecssc.

\section{OCTOBER 2005}

2-5 3rd Fracture and Flow in Advanced Glasses Conf.

University Park, PA. Carlo Pantano; ars2@psu.edu.

17-21 ASNT Fall Conf. and Quality Testing Show 2005,

Columbus, OH. Kelly Thomas, American Society for Nondestructive Testing, P.0. Box 28518, 1711 Arlingate Ln, Columbus, $\mathrm{OH} 43228-$ 0518; 800-222-2768 x227; fax 614274-6899; kthomas@asnt.org; http://www.asnt.org.

\section{NOVEMBER 2005}

14-16 $\nabla$ Continuous Casting of Non-Ferrous Metals Intl. Conf. \& Exhibition, Neu-UIm, Germany. Vera Hausen, Niels Parusel, DGM Secretariat, Deutsche Gesellschaft für Materialkunde, Hamburger Allee 26, Frankfurt, Germany, 49-69 7917-747; fax 49-69-7917-733; e-mail concast@dgm.de; www.dgm.de/concast.

22-25 Ninth NCB Intl. Seminar on Cement, New Delhi, India. NCCBM, Ninth NCB, 34 Km Stone, Delhi Mathura Road (NH2), Ballabgarh 121 004, Haryana, India, 91-129-2242051-56; fax 91-129-2242100, 2246175; e-mail nccbm@giasd101.vsnl.net.in.

29-3 MRS Fall Meeting Boston, MA. Materials Research Society, 506 Keystone Drive, Warrendale, PA 15086-7573; 724-779-3003; fax 724-779-8313; e-mail info@mrs.org www.mrs.org. MRS

\section{DECEMBER 2005}

5-7 2nd Intl. Conf. on Advances in Production and Processing of Aluminum (APPA 2005), Bahrain. Conf. Secretariat (APPA 2005), Univ. of Bahrain, P.0. Box 32038 , Bahrain, 973-17876114: fax 97317876655; e-mail appa2005@eng. uob.bh; www.uob.edu.bh/colleges/ engineering/conf/index.htm.

\section{MRS Future Meetings}

2005 SPRING MEETING

March 28-April 1

Exhibit: March 29-31

San Francisco, CA

2005 FALL MEETING

Nov. 28-Dec. 2

Exhibit: Nov. 29-Dec. 1

Boston, MA

2006 SPRING MEETING

April 17-21

Exhibit: April 18-20

San Francisco, CA

\section{Meeting Chairs:}

Joanna Aizenberg

Bell Labs

Lucent Technologies

jaizenberg@lucent.com

\section{Oliver Kraft}

University of Karlsruhe

oliver.kraft@imf.fzk.de
Neville R. Moody

Sandia National

Laboratories

nrmoody@sandia.gov
Ramamoorthy Ramesh

University of California

Berkeley

rramesh@uclink.berkeley.edu

Meeting Chairs:

\section{Yang-Tse Cheng}

General Motors R\&D Ctr.

yang.t.cheng@gm.com

David S. Ginley
National Renewable
Energy Laboratory
david_ginley@nrel.gov

Meeting Chairs:

Gregg S. Higashi

Applied Materials

gregg_s_higashi@amat.com
Viola Vogel
Swiss Federal Institute
of Technology, ETH

viola.vogel@mat.eth.eh
Kathryn E. Uhrich

Rutgers University

uhrich@rutchem.rutgers.edu

\section{Ralf B. Wehrspohn}

Paderborn University wehrspohn@physik. 